\title{
EDITORIAL
}

\section{THE UVEA: QUESTIONS OF PATHOGENESIS AND TREATMENT}

\begin{abstract}
Once again, the astonishing advances in ophthalmology during the last 25 years have made it necessary to expand a chapter in the Text-Book of Ophthalmology into a volume in this System. Many of our concepts of the ætiology of uveal disease have been changed during this period, and the treatment of many of them - although unfortunately not all - has been revolutionized. It is true that our ignorance of many things we would wish to know is still profound; but undoubtedly the present epoch in the development of medicine is exciting. Indeed, one of the most provocative and frustrating of the illnesses that affect the eye is uveitis, for the ætiology of most cases remains an enigma and, in the absence of a dramatic response to antibiotic drugs, we are often as impotent in our means of therapeusis as were our predecessors, apart from the somewhat cowardly but frequently very practical resource of blanketing the worst of the effects of inflammation by steroid drugs.
\end{abstract}

Sir Stewart Duke-Elder, Preface to System of Ophthalmology, vol. IX, Diseases of the Uveal Tract. C.V. Mosby, 1966.

Thirty years after Duke-Elder's statement, it is fair to state that we have made even more 'astonishing advances' in our understanding of the uveal tract and its disorders. Despite this progress, uveitis remains one of the most frustrating problems confronting ophthalmologists and their patients. It is therefore timely to focus the Twenty-Sixth Cambridge Ophthalmological Symposium on 'The Uvea'.

I will not review the anatomy, physiology, cell biology, etc., of the uvea; rather I will try to pose a series of questions from my own research - questions which have some bearing on the topics discussed during the symposium. These questions relate to our primate model of ocular histoplasmosis, which we developed in our effort to understand the human disease 'presumed ocular histoplasmosis syndrome' (POHS).

POHS was described in 1959 by Woods and Whalen, ${ }^{1}$ who observed haemorrhagic disciform macular disease with peripheral chorioretinal scars in patients with positive histoplasmin skin tests. The absence of vitreous or anterior segment inflammation and the presence of peripapillary scarring were clinical features of POHS later identified as part of the syndrome that has become a clinical entity in uveitis. $^{2}$ Despite the bulk of clinical and epidemiological data supporting an aetiological relationship between Histoplasma capsulatum and the characteristic ocular syndrome, some controversy remains as to the significance of this association. The precise aetiology of the late haemorrhagic macular disease, most often associated with the development of subretinal neovascularisation, also remains unknown. We developed our primate model in order to study these questions. ${ }^{3}$ As is often the case, however, our research resulted in more questions than answers. These questions have relevance to our understanding of other disorders of the uveal tract discussed during this symposium.

\section{Experimental Ocular Histoplasmosis Model ${ }^{3}$}

Non-human primates were given intracarotid injections of living $H$. capsulatum organisms. The injected primates developed an acute multifocal choroiditis, in which organisms and inflammatory cells were localised to the choroid (Fig. 1). The clinical choroiditis resolved spontaneously in 30-60 days; organisms could no longer be cultured or identified by microbiological, histopathological or immunohistochemical methods. The primates converted their histoplasmin skin tests to positive. Multiple healing patterns occurred over the months after injection, including typical choroioretinal scars or 'histo spots' in the periphery and around the posterior pole. However, some disappearing and/or clinically 'invisible' lesions occurred, which although clinically undetectable, consisted of active lymphocytic foci in the choroid with no organisms (Fig. 2). These foci of chronic choroiditis, present in both visible and clinically invisible lesions, persisted for years after the acute infection. 

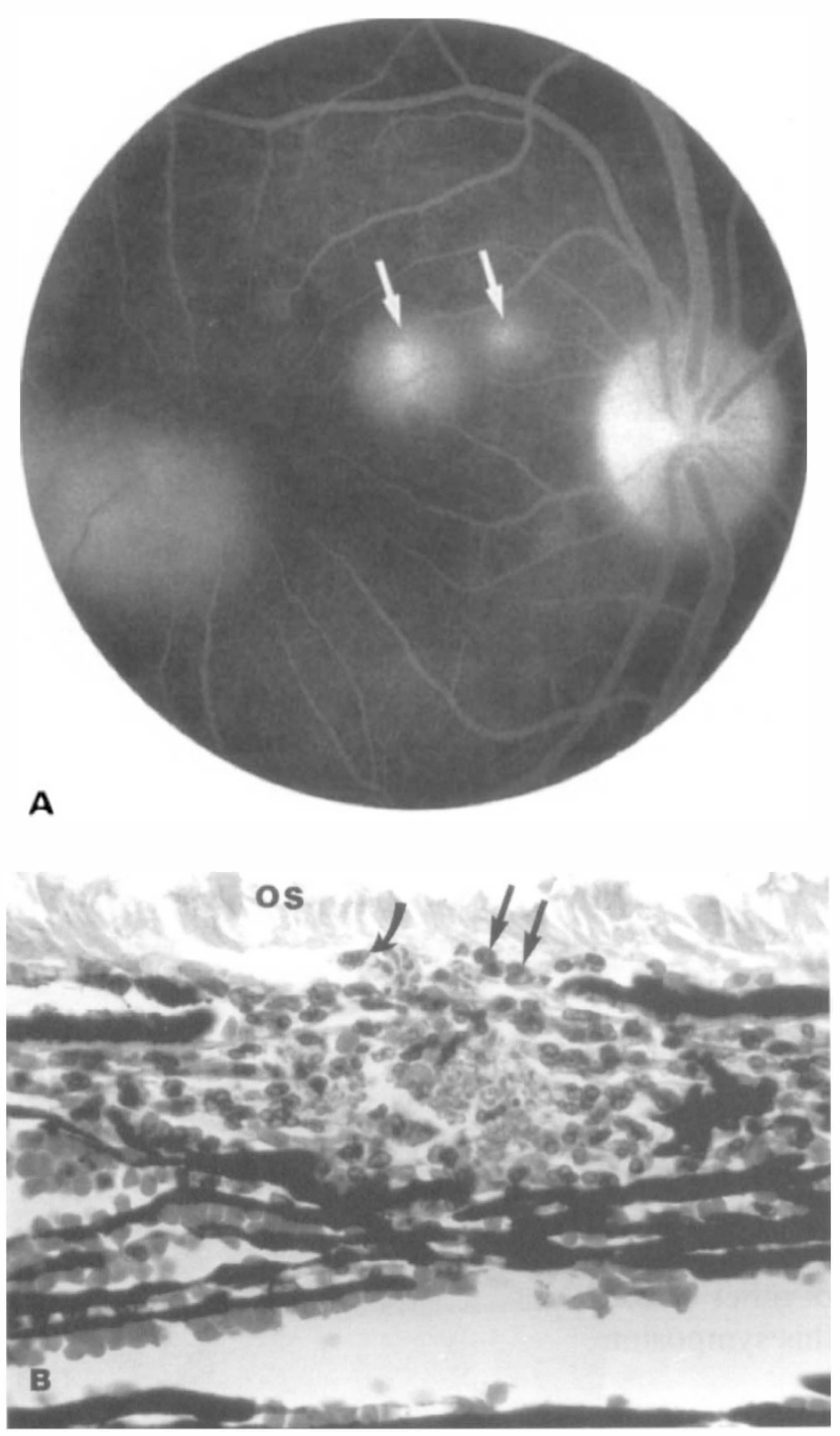

Fig. 1. Development of acute multifocal choroiditis in the non-human primate following intracarotid injection of live H. capsulatum. (A) Fluorescein angiography showing late staining of inflammatory lesions (arrows) 7 days after injection. (B) Histopathological finding in acute choroiditis demonstrating a break in Bruch's membrane with inflammatory cells (arrows) and what appears to be a cluster of organisms (curved arrow) penetrating into the subretinal space. $O S$, outer segment. $(H \& E, \times 600)$

At a time when the lesions were clinically 'inactive' (at least a year after the original acute multifocal choroiditis), intracarotid injections of killed organisms of $H$. capsulatum resulted in evidence of inflammatory reactivation in some eyes. ${ }^{3,4}$

\section{Why Do H. capsulatum Organisms Target the} Choroid?

It is not clear which factors determine the site of inflammation in various types of uveitis. In our model, is the size of the organism important? What about the size of the fenestrations in the chorio-
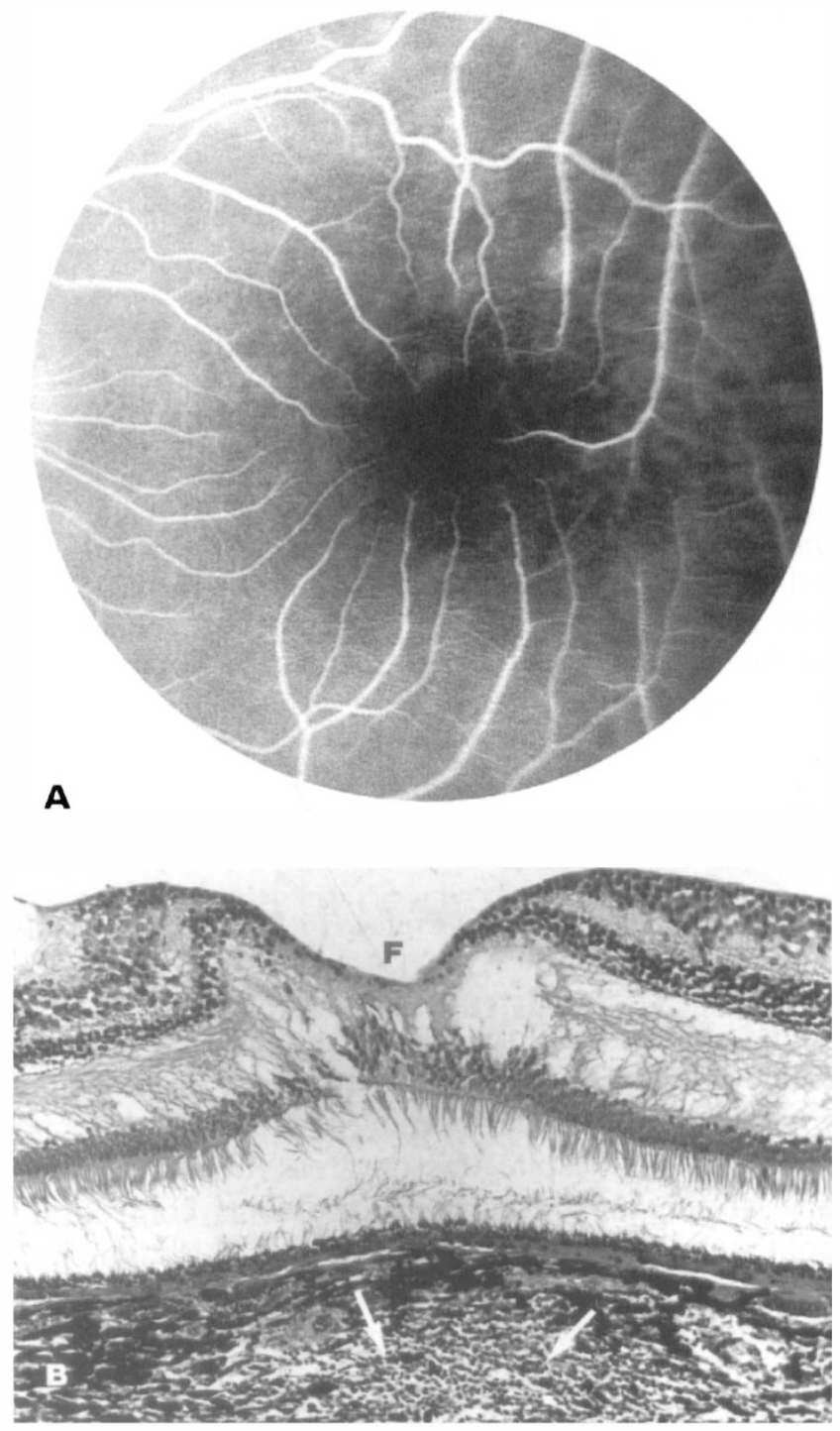

Fig. 2. Clinically invisible scar in a non-human primate 3 months after intracarotid injection of live $\mathrm{H}$. capsulatum. (A) Fluorescein angiography showing no visible staining in the fovea. (B) Histopathological evaluation showing large foci of mononuclear inflammatory cells (arrows) underlying what appears to be a normal retina, retinal pigment epithelium and choriocapillaris. $F$, fovea. $(H \& E, \times 180)$

capillaris? Is there some factor or factors which attract and hold organisms in the choroid?

Answers to these questions are not known. It is known that other organisms, such as Toxoplasma gondii, uniquely prefer the retina; and other syndromes such as sympathetic ophthalmia and VogtKoyanagi-Harada syndrome localise to the choroid.

What Factors Result in Neovascularisation in UveitisIncluding Subretinal Neovascularisation?

In human POHS, subretinal neovascularisation is part of the late macular disease, accounting for reduced vision when haemorrhage occurs. ${ }^{5}$

Defects in Bruch's membrane found in the primate model were apparently caused by direct damage due 
to the acute multifocal choroiditis. ${ }^{3}$ Although we did not observe the development of late haemorrhagic disciform lesions in the primate model, we did find histopathological evidence of defects in Bruch's membrane, with cells and vessels traversing Bruch's membrane into the subretinal space. This would seem to be the precursor of subretinal neovascularisation.

I believe that the sites of chronic choroidal lymphocytic infiltration observed in human peripheral histo scars and in our primate model ultimately result in damage to the Bruch's membrane/retinal pigment epithelium complex. These then become sites for subretinal neovascular membrane formation in some cases. There is not unequivocal evidence to support this theory, and neovascularisation occurs in a number of settings of ocular inflammation and uveitis. Factors leading to neovascularisation in uveitis are reviewed during the symposium. Are there particular cell types, molecules, proteins or factors which result in neovascularisation?

\section{What is the Nature and Significance of Chronic Clinical and Subclinical Choroiditis in Human and Experimental Ocular Histoplasmosis?}

In the primate model, acute histoplasmic choroiditis lesions resolved, leaving typical clinically detectable lesions. In some cases, however, there was no evidence of previous lesions, even by fluorescein angiography. ${ }^{3}$ The pathology in such eyes clearly showed the presence of foci of chronic choroiditis with no damage to the overlying retinal pigment epithelium in cases of 'invisible' choroiditis. No organisms were present. Similar foci of chronic choroiditis have been identified in histo spots from human POHS specimens, also without histopathological evidence of organisms. ${ }^{6-8}$ What stimulus keeps these inflammatory foci active in our model and in humans? No obvious organisms are present by culture or special stains. Are Histoplasma antigens present? If so, have we simply not yet been able to find them? We have not looked for these antigens by polymerase chain action (PCR) techniques; we have looked (unsuccessfully) with immunofluorescence techniques. $H$. capsulatum cell walls contain chitin, and chitinase is not present in humans. Therefore, histo cell wall remnants might remain in these choroidal lesions. Could other antigens such as altered retinal antigens or uveitogenic molecules be somehow associated with this model and present in the choroid itself? Does some form of immunoregulation control these inflammatory events? These questions are not unique to POHS and are important issues before this symposium.

What is the Pathogenesis of Apparent 'Reactivation' in Ocular Histoplasmosis?

'Reactivation' seemed to occur in the choroid after intracarotid injection of killed histo $H$. capsulatum organisms. It is not clear what mechanisms are involved in this process or in so-called reactivation in human POHS. Are these de novo lesions rather than reactivations? Most seem to occur in sites of previous acute multifocal choroiditis, but this has been difficult to document in every instance. It is my feeling that there is probably an immunopathological component to reactivation in this primate model, as evidenced by an apparent DTH response with increases in $\mathrm{CD}^{+}$lymphocytes and macrophages following antigen challenge. ${ }^{4}$ However, the model has not yet yielded definitive answers to this question.

Supported in part by NIH CORE grant EY03040 and an unrestricted grant from Research to Prevent Blindness, Inc., New York, New York.

Ronald E. Smith

Doheny Eye Institute, University of Southern California School of Medicine, Department of Ophthalmology, Los Angeles, California, USA

\section{References}

1. Woods AC, Wahlen HE. The probable role of benign histoplasmosis in the etiology of granulomatous uveitis. Trans Am Ophthalmol Soc 1959;57:318-43.

2. Schlaegel TF. Ocular histoplasmosis. New York: Grune \& Stratton, 1977:1-301.

3. Smith RE. Natural history and reactivation studies of experimental ocular histoplasmosis in a primate model. Trans Am Ophthalmol Soc 1982;80:695-757.

4. Palvolgyi I, Anderson A, Rife L, Taylor C, Smith RE. Immunopathology of reactivation of experimental ocular histoplasmosis. Exp Eye Res 1993;57:169-75.

5. Brown DM, Weingeist TA, Smith RE. Histoplasmosis. In: Pepose JS, Holland GN, Wilhelmus KR, editors. Ocular infection and immunity. St Louis: Mosby, 1996: 1252-62.

6. Irvine AR, Spencer WH, Hogan MJ, et al. Presumed chronic ocular histoplasmosis syndrome: clinical-pathologic case report. Trans Am Ophthalmol Soc 1976;94: 91-106.

7. Makley TA Jr, Craig EL, Long JW. Histopathology of presumed ocular histoplasmosis. Palestra Oftalmol Pan Am 1977;1:71-6.

8. Meredith TA, Green WR, Key SN III, et al. Ocular histoplasmosis: clinicopathologic correlation of 3 cases. Surv Ophthalmol 1977;22:189-205. 